\title{
Corticoesteroides y cirugía del tercer molar inferior. Revisión de la literatura
}

\author{
Prieto I*, Prieto-Fechech A**, Bascones Martínez A***
}

\section{RESUMEN}

El objetivo de este trabajo es buscar la evidencia disponible con respecto al uso, justificación, efectos secundarios y eficacia de corticosteroides para reducir el dolor e inflamación postoperatorios en la extracción quirúrgica de terceros molares. Queremos saber si en pacientes adultos, es la prescripción de antiinflamatorios esteroideos además de los antiinflamatorios no esteroideos (AINES), en comparación con la administración única de AINES, más efectiva para mejorar, aliviar o eliminar los síntomas de inflamación y dolor después de la extracción quirúrgica de terceros molares inferiores. En la revisión de la literatura se ha utilizando la base de datos Medline con las palabras clave "esteroides" y "terceros molares" o "Muelas del juicio" y "Cirugía". La búsqueda fue limitada también a estudios en humanos, lengua inglesa y revistas dentales.

Palabras clave: "Esteroides", "Terceros Molares", "Muelas del Juicio", "Cirugía”

\section{ABSTRACT}

The aim of this study is searching the available evidence with respect to the use, justification, side effects and efficacy of corticosteroids in reducing pain and swelling after the surgical extraction of impacted lower third molars. We want to investigate if steroidal anti-inflammatories prescription in addition to non-steroidal anti-inflammatory drugs (NSAID), when compared with the exclusive administration of NSAID, is more effective improving, relieving or eradicating symptoms of swelling and pain after the extraction of the impacted lower third molar. We have utilize in this literature review the Medline database with the keywords "steroids" and "Third molars" or "wisdom molars" and "surgery". The search was also limited to humans, English and dental journals.

Key words: "Steroids", "Third Molars", "Wisdom Teeth", "Surgery”

Aceptado para publicación: Abril 2005.

* Doctor en Odontología por la UCM. Experto en "Periodoncia Clínica". Alumno del Master de Periodoncia de la UCM.

** Cirujano Maxilofacial. Hospital Ramón y Cajal. Jefe de Sección de Servicio de Cirugía.

*** Doctor en Medicina y Cirugía por la UCM. Doctor en Estomatología por la UCM. Catedrático de Periodoncia y Patología Bucal de la UCM.

Prieto I, Prieto-Fenech A, Bascones Martínez A. Corticoesteroides y cirugía del tercer molar inferior. Revisión de la literatura. Av. Odontoestomatol 2005; 21-5: 251-258. 


\section{MATERIAL Y MÉTODOS}

El objetivo de esta revisión sistemática sobre el papel de los corticoesteroides para reducir el dolor, la inflamación y trismus postoperatorios en la extracción quirúrgica de terceros molares, fue examinar los ensayos clínicos relacionados con el tratamiento farmacológico de pacientes que van a ser sometidos a dicha intervención.

En la revisión de la literatura se ha utilizando la base de datos Medline con las palabras clave "esteroides" y "terceros molares" o "Muelas del juicio" y "Cirugía". La búsqueda fue limitada también a estudios en humanos, lengua inglesa y revistas dentales.

Se encontraron 128 artículos, relacionados con las palabras clave mencionadas, de los cuales, mediante los títulos, pudimos rechazar 78 estudios sin interés para el objetivo de este trabajo. Posteriormente, mediante la lectura de los resúmenes, pudimos hacer una selección más fina incluyendo, finalmente 16 estudios fueron incluidos y discutidos en nuestra revisión.

\section{INTRODUCCIÓN}

La extracción quirúrgica de los terceros molares, hoy en día es un procedimiento frecuente en el campo de la cirugía oral. Es una práctica no exenta de complicaciones, mecánicas, nerviosas, inflamatorias e infecciosas. La extracción quirúrgica de los terceros molares produce casi siempre un cuadro inflamatorio agudo que genera una intensa sensación de discomfort. A pesar del tratamiento con AINES o corticoides, administrado pre, intra o post-quirúrgicamente, el proceso inflamatorio aparece en la gran mayoría de postoperatorios inmediatos. La agresión a los tejidos blandos orales y periorales, el despegamiento del colgajo y el traumatismo óseo, son los responsables de la inflamación que, en condiciones normales, se intensifica presentando un pico a las 72 horas. A partir de aquí comienza a remitir, y decrece durante otras 48 o 72 más. Para combatir sus posibles complicaciones se han propuesto varias combinaciones farmacológicas y algunos procedimientos técnicos que intentan paliar las secuelas indeseables de esta cirugía.(1) Es obvio que el mejor tratamiento preventivo de la inflamación consiste en la realización de una cirugía técnicamente correcta, rápida, limpia y lo más atraumática posible, aplicando los productos más inocuos y efectivos que nos brinda la farmacología.

Los corticoides tienen un potente efecto inhibidor de la inflamación, y han sido utilizados con distintas pautas y vías para disminuir las secuelas inflamatorias de la exodoncia quirúrgica del tercer molar. Inducen la síntesis de proteinas endógenas, las cuales bloquean la actividad enzimática de la fosfolipasa A2. El bloqueo en este punto inhibe la liberación del ác. araquidónico por los constituyentes de la membrana celular, impidiendo finalmente la síntesis de prostaglandinas, leucotrienos, o sustancias relacionadas con el tromboxano. Estos efectos son la base

\begin{tabular}{|lllll|}
\hline \multicolumn{4}{c}{ TABLA 1.- CORTICOIDES REPRESENTATIVOS* } \\
\hline Esteroide & $\begin{array}{l}\text { Potencia Relativa } \\
\text { Antiinflamatoria }\end{array}$ & Retención Na+ & $\begin{array}{l}\text { Semivida } \\
\text { Biológica (Horas) }\end{array}$ & $\begin{array}{l}\text { Dosis Supresora } \\
\text { de ACTH (mg/d) }\end{array}$ \\
\hline Cortisol & 1 & 1 & $8-12$ & $20-30$ \\
Cortisona & 0,8 & 0,8 & $8-12$ & $25-35$ \\
Prednisolona & 4 & 0,8 & $12-36$ & $7,5-10$ \\
Prednisona & 4 & 0,8 & $12-36$ & $7,5-10$ \\
Triamcinolona & 5 & 0 & $18-36$ & $7,5-10$ \\
Parametasona & 10 & 0 & $24-48$ & 5 \\
Dexametasona & 30 & 0 & $24-72$ & 1,5 \\
Betametasona & 30 & 0 & $24-72$ & 1,5 \\
Fludrocortisona & 10 & 250 & $8-12$ & $2,5-5$ \\
\hline * Modificada de Bascones y col.(3) & \multicolumn{3}{l}{} \\
\hline
\end{tabular}


de la utilización clínica, pero también son responsables de algunos de los graves efectos producidos por la administración crónica de corticoides. (2) Efectivamente, los glucocorticoides inhiben la liberación de ACTH, lo que tras un uso continuado conlleva una atrofia del tejido suprarrenal. (3)

La farmacología actual dispone de corticoides con un potencial antiinflamatorio variable, como se puede valorar en la tabla 1 .

\section{REVISIONES}

Barron y cols. en 2004(2) realizaron un estudio preliminar para valorar el efecto de la dexametasona (Dx) y la dipirona (derivado pirazolónico con efecto fundamentalmente analgésico), en la hipersensibilidad del nervio dentario después de la extracción de terceros molares. El estudio trata de valorar mediante un test sensorial cuantitativo electrónico, los efectos de esos fármacos en los cambios sensoriales del territorio de inervación del nervio dentario, del nervio lingual y de la región del nervio infraorbitario, antes de la cirugía así como dos y ocho días después de la exodoncia del tercer molar inferior. Toman 14 pacientes divididos en dos grupos, a unos les administraron Dx, (8 mg. Oral 30' preoperatoriamente) y dipirona (1g. vía oral 30 mín. antes de la cirugía) y en el grupo control sólo dipirona con la misma pauta. En ambos grupos se administra además tratamiento antibiótico. Como resultado se obtiene que los pacientes que reciben Dx, presentan unos niveles de sensibilidad nerviosa postoperatoria, en los nervios lingual y dentario inferior, igual a los valores basales, mientras que los que reciben sólo dipirona obtuvieron reducciones significativas en los umbrales de detección eléctrica de dichos nervios. Los autores concluyen que el tratamiento con la asociación de estos dos fármacos previene la aparición de la hipersensibilidad postextracción de terceros molares inferiores. Se trata de un modo bastante objetivo para valorar la efectividad del medicamento y es exportable para investigaciones con otros esquemas terapéuticos.

Dionne y cols.(4) en 2003 evalúan in vivo los niveles de prostanoides (mediadores en la respuesta infla- matoria de los glucocorticoides) en el sitio de la lesión mediante una microsonda instalada submucogingival por bucal en el momento de la cirugía. Por otro lado, se mide el dolor mediante una escala analógica visual. Se estudian 61 pacientes en dos grupos randomizados que reciben placebo o la Dx administrada en una dosis de $4 \mathrm{mg}$ por vía oral 12 horas antes y $4 \mathrm{mg}$ por vía oral una hora antes de la cirugía de los terceros molares. Los pacientes seleccionados con estatus Clase I de anestesia, de 18 a 35 años con indicación de extracción de los dos cordales inferiores parcial o totalmente impactados. Después de la cirugía, cuando aparecía el dolor, se administraban iv. 30 mg. de Ketorolako (potente inhibidor de las vias COX1 y COX2 del ácido Araquidónico y por tanto analgésico de acción periférica), o bien placebo. La prostaglandina PGE2 fue detectada, mediante la microsonda, en el sitio de la cirugía. Disminuyó durante la hora siguiente para después incrementarse en relación con la aparición del dolor. El Ketorolaco disminuye significativamente los niveles de PGE2 cuando se produce dolor en comparación con las muestras de placebo y la TXB2. Los autores encuentran que la Dx carece de efecto analgésico, en comparación con el ketorolaco que produce reducción mas profunda de los niveles de PGE2 y TXB2. Sugieren que los glucocorticoides a esta dosis, no reducen en cantidad suficiente los niveles de prostaglandinas para atenuar la sensibilización periférica de los nociceptores después del trauma operatorio. Estos autores, considerando la eficacia de los AINES y los analgésicos, no apoyan la utilización de los corticoides para el tratamiento del dolor en la extracción quirúrgica de los terceros molares aunque reconocen el efecto antiinflamatorio de la Dx.

Neupert y cols.(5) en 1992 evaluaron el tratamiento con Dx para la reducción de las secuelas postoperatorias de la exodoncia quirúrgica de terceros molares. Llevaron a cabo un estudio a doble ciego con 60 pacientes con dosis de $4 \mathrm{mg}$. de Dx. Los pacientes fueron citados para las cirugías en el lado test y control con 5 ó 6 semanas de separación, aplicando aleatoriamente una dosis de Dx iv. de $4 \mathrm{mg}$. preoperatoria, o bien agua estéril en el lado control. Se verificó el dolor mediante escala analógica, el trismus por medición interincisal y la inflamación midiendo la distancia entre puntos faciales predeterminados. No 
se apreciaron diferencias en el dolor diario ni en la inflamación, sin embargo el dolor global y el trismus fue significativamente afectado por el esteroide, obteniéndose un incremento diario postoperatorio de abertura máxima interincisal de 4 a $6 \mathrm{~mm}$. con respecto al lado control y los pacientes escogieron con un ratio de 4:1 el lado del esteroide como el menos doloroso. Además los autores no observaron complicaciones ni mayores ni distintas en el lado de los esteroides con respecto al lado control. Contrasta relativamente con Dionne y cols.(4), ya que no evidencian disminución del dolor ni de la inflamación, aunque si del trismus y del dolor global subjetivo por lo que los pacientes están más satisfechos con la evolución del cordal tratado con corticoides. En la discusión de este articulo por el Dr. Ross Beirne(6), se pregunta porqué si casi todos los estudios sobre el tema los corticoides reducen significativamente el dolor, la inflamación y el trismus, en el estudio no son capaces de demostrar estos efectos $y$, afirma que es importante resolverlo, porque los corticoides pueden presentar importantes efectos secundarios y no deben ser utilizados si no son efectivos. Los estudios que han demostrado eficacia de los corticoides utilizan dosis de $125 \mathrm{mg}$. de MP, equivalente a $625 \mathrm{mg}$. de hidrocortisona, mientras que 4 mg. de Dx equivalen a 106 mg de hidrocortisona. Hay dos estudios previos que con dosis de $9 \mathrm{mg}$. por vía oral y otro intramuscular de $8 \mathrm{mg}$. no consiguen reducir el dolor, luego es evidente que estos autores han utilizado unas dosis subterapéuticas.

En 1985 Pedersen(7) investiga el efecto preventivo de $4 \mathrm{mg}$ de Dx sobre el dolor, trismus e inflamación, después de la exodoncia quirúrgica de terceros molares impactados en 30 individuos sanos. Cada paciente sirvió como autocontrol al recibir una inyección en el masetero antes del comienzo de la cirugía o bien del esteroide, o bien del placebo en el sitio contrario. Se realizaron controles a las 48 horas y a la semana. El dolor fue evaluado mediante una escala visual analógica y por el consumo de comprimidos de $500 \mathrm{mg}$. de paracetamol. El tamaño de la inflamación postoperatoria fue determinado por estereofotografía y la apertura bucal registrada mediante la medición de la distancia interincisal. Según los datos de este estudio la administración de corticoides reduce un $49 \%$ de la inflamación postoperatoria y del trismus, así como el $30 \%$ del dolor postoperatorio.
Los autores no refieren complicaciones locales ni generales y concluyen con que el tratamiento profiláctico con esteroides es efectivo para la reducción de las molestias postoperatorias y la administración es segura en ausencia de contraindicaciones. Se recomienda el tratamiento profiláctico en la cirugía del tercer molar cuando se esperen reacciones postoperatorias pronunciadas.

En 1985 Elhag y cols.(8) realizan un ensayo controlado a simple ciego para determinar el efecto antiinflamatorio de $10 \mathrm{mg}$ de Dx administrados pre y postoperatoriamente en comparación con el tratamiento con ultrasonidos en pacientes operados de terceros molares inferiores impactados. La inflamación facial medida con un edemómetro, y el trismus fueron significativamente reducidos en ambos grupos comparados con otro grupo control no tratado. Este trabajo controla los niveles de cortisol en plasma para evaluar la afectación secundaría del eje hipotálamo hipofisiario obteniendo niveles normales a los tres días después de una depresión significativa 24 horas después de la administración de Dx.

En 1975 Messer y cols.(9) evaluaron el uso de la Dx intraoral por vía intramuscular directamente sobre el masétero, después de la extracción de terceros molares mandibulares. Todos los cirujanos orales del estudio encontraron problemas de dolor, inflamación y trismus postoperatorio. Estas complicaciones a menudo son directamente proporcionales al edema. Por lo tanto en pacientes con mínimo edema, el dolor y el trismus deben ser proporcionalmente reducidos. Estos autores, en el estudio realizado, hacen una valoración clínica de los corticoides utilizados en más de 5000 pacientes y apoyan el uso de la Dx intramaseterina para aliviar el edema y aliviar el edema postoperatorio. No obstante, ha de tenerse en cuenta que el estudio de Messer esta basado en la experiencia clínica.

En 2003, Üstün y cols.(10) estudian la influencia de la dosis de MP en el dolor, la inflamación y el trismus después de la extracción de terceros molares. Comparan el efecto de 1,5 y $3 \mathrm{mg}$./Kg de peso de MP en los síntomas postoperatorios de extracción de terceros molares. El diseño consistió en un estudio transversal a doble ciego con 26 pacientes sanos con cordales simétricos impactados mandibulares. 
En la cirugía de un lado reciben la dosis de 1,5 $\mathrm{mg} . / \mathrm{kg}$. de peso intravenosa una hora antes, y en la cirugía contralateral reciben el doble de dosis también una hora antes. Utilizaron una escala analógica para determinar el dolor de los pacientes y también valoraron el trismus y la inflamación. Los autores no encuentran diferencias significativas y por tanto concluyen que no son necesarias dosis más altas de MP para obtener el mismo resultado. En este caso si utilizan métodos objetivos para la valoración del trismos y del dolor.

En 1999 Esen y cols.(11) mediante un estudio a doble ciego determinaron el efecto antiinflamatorio y la supresión adrenal de la MP (MP) en las secuelas de la cirugía de los terceros molares. Cada uno de los 20 pacientes que iban a someterse a la cirugía de terceros molares simétricos inferiores recibió preoperatoriamente $125 \mathrm{mg}$. de MP intravenosamente y un placebo en la otra cirugía de manera aleatoria. Se determinó la inflamación y el edema facial, el trismo y el dolor, que se valoró registrando el número de tabletas analgésicas precisadas el día de la cirugía y el siguiente. La función del eje hipotalámico-pituitario-adrenal fue determinada por la medición de la concentración plasmática basal de cortisol pre y postoperatoria. También se realizó un test de estimulación de la hormona adrenocorticotropa (ACTH), antes y después de la administración de MP para evaluar la función adrenal. El análisis estadístico determinó un significativo descenso de los niveles de edema, trismus y dolor en el grupo de MP. Los niveles de cortisol en sangre experimentaron variaciones insignificantes entre ambos grupos y el test de la ACTH, demostró función normal antes y después de la administración de MP. No se apreció otro tipo de alteración evolutiva, infección, cicatrización etc. en el grupo MP. El 90\% de los pacientes manifestaron su preferencia por el tratamiento con MP. Los autores concluyeron que en ausencia de contraindicaciones, el uso iv preoperatorio de $125 \mathrm{mg}$ de MP es un método efectivo y seguro para prevenir las complicaciones postoperatorias de la cirugía de los terceros molares.

Schultze y cols.(12) en 1995 testaron el uso combinado de ibuprofeno y MP para la prevención del dolor e inflamación después de la extracción de terceros molares impactados superiores e inferiores.
Para ello, en su estudio a doble ciego administraron en un lado $16 \mathrm{mg}$. de MP 12 horas antes y 12 horas después de la cirugía y además 400 mg. de ibuprofeno cada ocho horas en el día de la operación y los dos días siguientes. En el otro lado de control sólo se administró placebo. El examen ultrasónico demostró una significativa reducción de la inflamación del 56\% comparada con el grupo placebo. Medida con cinta métrica, la reducción fue del $58 \%$. La escala visual de reducción del dolor fue del $67 \%$. La conclusión de los autores fue que la combinación de la MP con ibuprofeno tuvo un buen efecto antiinflamatorio y analgésico. Otra conclusión extraída por los autores, es que la combinación de estos medicamentos tiene una acción sinérgica. Aunque es probable que esto sea así, según Hargreaves y cols.(13), con este estudio no se demuestra que la combinación de los dos medicamentos potencie el efecto antiinflamatorio. Hargreaves también sugiere que el descubrimiento del mecanismo de acción de los glucocorticóides cuya acción depende de la síntesis de proteínas más que de la estabilización de la membrana celular, requiere un tiempo de una a cuatro horas para que se inicie el efecto antiinflamatorio, por lo que no considera necesario administrar la dosis previa tan anticipadamente.

En 1993 Hyrkas y cols.(14) compararon el uso de diclofenaco con y sin $40 \mathrm{mg}$. de MP intravenoso para prevenir el dolor postoperatorio después de la extracción del tercer molar, y concluyeron que la eficacia de la administración preoperatoria intravenosa de $40 \mathrm{mg}$ de MP en combinación con diclofenaco de liberación rápida y de liberación sostenida, es más eficaz que el diclofenaco sólo. Sin embargo la duración del efecto paliativo del dolor en el grupo test en este estudio sólo duró cuatro horas.

Troullos y cols.(15) en 1990 comparan mediante dos estudios contrastados la influencia del uso del ibuprofeno y del flurbiprofeno en combinación con MP y placebo en el dolor agudo, trismus e inflamación posteriores a la exodoncia quirúrgica de terceros molares. Los resultados del estudio indican que los AINES producen una mayor analgesia inicial que los esteroides, sin embargo, estos son más potentes a la hora de reducir la cantidad de inflamación y útiles para evitar la pérdida de función. Los AINES preoperatorios, previenen sólo modestamente la inflamación en com- 
paración con el placebo. Los autores sugieren que el efecto analgésico de los AINES (en el modelo de la cirugía oral) es debido, más que al efecto antiinflamatorio generalizado, a la supresión de los mediadores bioquímicos del proceso de nocicepción (prostaglandinas). Aunque no se evaluó en esta investigación, parece ser que la máxima analgesia, antiedema, y preservación de la función, sería obtenida por medio de la combinación de AINES y corticoides.

En 1986 Beirne y cols.(16), valoraron el efecto de la MP sobre el dolor, trismus e inflamación después de la extracción de terceros molares. Diseñaron un estudio con 31 individuos divididos aleatoriamente en dos grupos. El primero con 16 pacientes, recibe, antes de la sedación, suero salino en solución y el otro, de 15 individuos, $125 \mathrm{mg}$ de MP intravenosa. Se midió la inflamación por técnica fotográfica, el trismus por medición interincisal y el dolor mediante registro del número de píldoras analgésicas precisadas. El dolor y la inflamación fueron significativamente reducidos por la administración de una dosis de MP, y el trismus también era menor en el grupo de MP pero no significativamente menor. Una conclusión que se puede sacar de este estudio es que una dosis de MP por sí sola es eficaz el primer día, pero a partir del segundo día se igualan los parámetros medidos, probablemente debido al rápido metabolismo de este medicamento. Esto refuerza la necesidad de combinar los corticoides con AINES.

En 1985 Bystedt (17) realiza un estudio transversal, a boca partida y a doble ciego en el que valora el efecto de la MP después de la extracción de terceros molares impactados. Con este estudio, en el que participaron 20 pacientes sanos que sufrieron la exodoncia quirúrgica de sus terceros molares, se vio una disminución de las molestias postoperatorias en cuanto a trismus e inflamación. Sin embargo los autores no encuentran diferencias estadísticamente significativas con el grupo placebo.

En 1985 Sisk y cols.(18) evaluaron la eficacia de los corticoides, aines y placebo en la reducción de la respuesta inflamatoria aguda postoperatoria y en las secuelas indeseables producto de las extracciones quirúrgicas de los terceros molares, y para ello utilizaron en su ensayo MP, flurbiprofeno y placebo. Según estos autores los corticoides parecen tener el máximo efecto en el control del edema pero un mínimo efecto analgésico. Los aines parece que son efectivos como analgésicos. Recomiendan el uso combinado de estos agentes para el control de las secuelas de estos procedimientos de cirugía oral.

Fridrich y cols. (19) en 1990 evalúan dos métodos que pueden ser universalmente utilizados para prevenir la incidencia de alveolitis. El diseño del estudio es a boca partida en pacientes con cordales simétricos. Se estudian otras variables como el sexo, aspirina previa a la exodoncia, edad, anticonceptivos etc., en relación con la evolución postoperatoria. Para este proyecto se basan en 952 extracciones a 476 pacientes, evaluando apósitos de lincomicina en gelfoam (esponja de gelatina) reabsorbible frente a terra-cortryl en gelfoam y frente a gelfoam con suero salino sólo. Las dos primeras combinaciones fueron efectivas, no siéndolo la esponja sola con suero. La edad y los anticonceptivos orales no influyeron en el resultado.

Gersema y cols.(20) en 1992 revisaron 10 ensayos clínicos en relación con el uso de corticoides perioperatorios en cirugía oral para la reducción del dolor, trismus y edema postquirúrgico. Analizaron el tipo de procedimiento, el régimen específico, su potencia relativa y los métodos utilizados para valorar los resultados. Según los autores, los primeros ensayos revisados demuestran subjetivamente que los corticoesteroides reducen la cantidad de inflamación asociada con cirugía oral, especialmente el edema, sin embargo posteriores evaluaciones más objetivas si demuestran que el uso de corticoides reducen consistentemente el edema. Según esta revisión las dosis de corticoesteroides empleadas en los estudios fluctuaron de 80 a $625 \mathrm{mg}$. de su equivalente con hidrocortisona sin observarse en ningún caso reacciones adversas. Estos autores concluyen que el uso de los corticoides en la cirugía de los terceros molares es un método seguro y racional para la reducción de las complicaciones postoperatorias.

\section{DISCUSIÓN Y CONCLUSIONES}

La conclusión que podemos extraer de este trabajo de revisión señala que los corticoides son más efec- 
tivos para eliminar los síntomas de inflamación y trismus y los AINES son más bien efectivos como analgésicos. La dexametasona y la metilprednisolona son los corticoides más utilizados. Ahora bien, la combinación de corticoides con AINES, según la bibliografía revisada, mejora significativamente las secuelas postoperatorias en la exodoncia quirúrgica de terceros molares. No obstante, son medicamentos con efectos secundarios a tener en cuenta y contraindicaciones que el clínico debe conocer. Teniendo en cuenta las características individuales de cada paciente y de cada cordal, no parece conveniente establecer unas recomendaciones rígidas, pero es esperable mas inflamación, dolor y trismus cuanto mas despegamiento, mas osteotomía, mas manipulación, mas intentos de avulsión, y en general mas duración de la operación. Por lo tanto, se impone un tratamiento adecuado a cada situación. Además parece que no hay relación entre el volumen de la inflamación y la cantidad de dolor.

En general se comunican pocas complicaciones sépticas, sin embargo teniendo en cuenta que la inflamación es un mecanismo fisiológico necesario, su disminución va a constituir un factor de riesgo objetivo en la aparición de la misma, por lo que sería prudente, y de hecho se hace casi siempre así, complementar con antibioterapia.

Aunque no se demuestra el efecto sinérgico con los AINES, su efecto analgésico es más importante que el obtenido por los esteroides y se recomiendan unas dosis más bajas y más frecuentes de ibuprofeno cuando se indica como complemento terapéutico.

No hemos encontrado investigaciones sobre los preparados a base de acetato de betametasona en cirugía oral. Esta fórmula permite una liberación lenta y por tanto supera la fugacidad de acción de los corticoides hidrosolubles. Existen en el mercado preparados que combinan fosfato y acetato de betametasona que permiten unos niveles terapéuticos mantenidos.

\section{BIBLIOGRAFIA}

1. Sada JM. Cordales Incluidos. En: Bascones A, editor. Tratado de Odontología. Madrid:
Ediciones Avances Médico-Dentales, S.L.; 1999. p. 3643-53.

2. Barron RP, Benoliel R, Zeltser R, Eliav E, Nahlieli $\mathrm{O}$, Gracely $\mathrm{RH}$. Effect of dexamethasone and dipyrone on lingual and inferior alveolar nerve hypersensitivity following third molar extractions: preliminary report. J Orofac Pain 2004;18(1):628.

3. Quintana LA, Raczka WE. Farmacología del Sistéma Endocrino: Bases Farmacológicas. En: Bascones A, Bullon P, Castillo JR, Machuca G, Manso FJ, Serrano JS, editors. Bases Farmacológicas de la Terapéútica Odontológica. Madrid: Avances; 2000. p. 175-208.

4. Dionne RA, Gordon SM, Rowan J, Kent A, Brahim JS. Dexamethasone suppresses peripheral prostanoid levels without analgesia in a clinical model of acute inflammation. J Oral Maxillofac Surg 2003 Sep;61(9):997-1003.

5. Neupert EA, III, Lee JW, Philput CB, Gordon JR. Evaluation of dexamethasone for reduction of postsurgical sequelae of third molar removal. J Oral Maxillofac Surg 1992 Nov;50(11):1177-82.

6. Beirne OR. Discussion: Evaluation of dexamethasone for reduction of postsurgical sequelae of third molar removal. J Oral Maxillofac Surg 1992 Nov;50(11):1182-3.

7. Pedersen A. Decadronphosphate in the relief of complaints after third molar surgery. A doubleblind, controlled trial with bilateral oral surgery. Int J Oral Surg 1985 Jun;14(3):235-40.

8, ElHag M, Coghlan K, Christmas P, Harvey W, Harris $M$. The anti-inflammatory effects of dexamethasone and therapeutic ultrasound in oral surgery. Br J Oral Maxillofac Surg 1985 Feb;23(1):17-23.

9. Messer EJ, Keller JJ. The use of intraoral dexamethasone after extraction of mandibular third molars. Oral Surg Oral Med Oral Pathol 1975 Nov;40(5):594-8. 
10. UStun Y, Erdogan O, Esen E, Karsli ED Comparison of the effects of 2 doses of methylprednisolone on pain, swelling, and trismus after third molar surgery. Oral Surg Oral Med Oral Pathol Oral Radiol Endod 2003 Nov;96(5):535-9.

11. Esen E, Tasar F, Akhan O. Determination of the anti-inflammatory effects of methylprednisolone on the sequelae of third molar surgery. $\mathrm{J}$ Oral Maxillofac Surg 1999 Oct;57(10):1201-6.

12. Schultze-Mosgau S, Schmelzeisen R, Frolich JC, Schmele H. Use of ibuprofen and methylprednisolone for the prevention of pain and swelling after removal of impacted third molars. J Oral Maxillofac Surg 1995 Jan;53(1):2-7.

13. Hargreaves KM. Discussion : Use of ibuprofen and methylprednisolone for the prevention of pain and swelling after removal of impacted third molars. Journal of Oral and Maxillofacial Surgery 1995 Jan;53(1):7-8.

14. Hyrkas T, Ylipaavalniemi P, Oikarinen VJ, Paakkari I. A comparison of diclofenac with and without single-dose intravenous steroid to prevent postoperative pain after third molar removal. J Oral Maxillofac Surg 1993 Jun;51(6):634-6.
15. Troullos ES, Hargreaves KM, Butler DP, Dionne RA. Comparison of nonsteroidal anti-inflammatory drugs, ibuprofen and flurbiprofen, with methylprednisolone and placebo for acute pain, swelling, and trismus. J Oral Maxillofac Surg 1990 Sep;48(9):945-52.

16. Beirne OR, Hollander B. The effect of methylprednisolone on pain, trismus, and swelling after removal of third molars. Oral Surg Oral Med Oral Pathol 1986 Feb;61(2):134-8.

17. Bystedt H, Nordenram A. Effect of methylprednisolone on complications after removal of impacted mandibular third molars. Swed Dent J 1985;9(2):65-9.

18. Sisk AL, Bonnington GJ. Evaluation of methylprednisolone and flurbiprofen for inhibition of the postoperative inflammatory response. Oral Surg Oral Med Oral Pathol 1985 Aug;60(2):137-45.

19. Fridrich KL, Olson RA. Alveolar osteitis following surgical removal of mandibular third molars. Anesth Prog 1990 Jan;37(1):32-41.

20. Gersema L, Baker K. Use of corticosteroids in oral surgery. J Oral Maxillofac Surg 1992 Mar;50(3):270-7. 